\title{
Effect of Positive Psychotherapy Based on Virtual Social Networks on Anxiety and Depression Reduction in Veterans with Spinal Cord Injury
}

\section{ART I C L E INF O}

\section{Article Type}

Original Research

\section{Authors}

Firoozy M.* $P h D$

Ramezani Piyani M. ${ }^{1} M S c$

How to cite this article
Firoozy M, Ramezani Piyani M.
Effect of Positive Psychotherapy
Based on Virtual Social Networks
on Anxiety and Depression Red-
uction in Veterans with Spinal
Cord Injury. Iranian Journal of
War \& Public Health. 2018;10
(1):21-26.

*Clinical Psychology Department, College of Farabi, University of Tehran, Tehran, Iran

${ }^{1}$ Clinical Psychology Department, College of Farabi, University of Tehran, Tehran, Iran

\section{Correspondence}

Address: Clinical Psychology Department, College of Farabi, University of Tehran, Enghelab Street, Tehran, Iran

Phone: +98 (025) 36166650

Fax: +98 (025) 36644081

mfiroozy@ut.ac.ir

\section{Article History}

Received: August 05, 2017

Accepted: October 17, 2017

ePublished: January 11, 2018

\section{A B S T R A C T}

Aims Spinal cord injured veterans' problems significantly affect on psychological health of these people. Because of veterans' disability to move and, consequently, excessive difficulty for going to health centers and attending therapeutic sessions, makes it necessary to carry out remote psychological interventions. Therefore, the purpose of this study was to investigate the effectiveness of positive psychology on decreasing psychological distress among veterans through virtual social networks.

Materials \& Methods The present applied research is a semi-experimental study with pretest and post-test design with control group that carried out among 38 veterans with spinal cord injury in Khuzestan province. They were randomly selected from the list of Martyr and Sacrifices' Foundation of Khuzestan province in 2017 and they were randomly assigned into experimental and control groups (19 people in each group). For data collection, Beck Depression Inventory (BDI-II) and Beck Anxiety Inventory were used in pre-test and post-test. The experimental group received 14 sessions of positive psychotherapy and the control group did not receive any interventions. Data were analyzed by SPSS 22 software using Analysis of Covariance (ANCOVA).

Findings There was a significant difference in post-test scores of depression and anxiety between experimental and control groups and positive psychological intervention significantly reduced depression and anxiety in the experimental group $(\mathrm{p}=0.001)$.

Conclusion Positive psychological interventions based on virtual social networks (telegrams) is effective on anxiety and depression reduction in veterans with spinal cord injury.

Keywords Veteran; Spinal Cord Injury; Social Networks; Mental Health

\section{CIT A T I O N L INKS}

[1] Predicting trait competitive anxiety, competitive aggression, and competitive anger based on spiritual intelligence ... [2] Effectiveness of emotion focused therapy on emotional dysregulation, hopelessness ... [3] International differences in ageing and ... [4] Positive ... [5] Positive psychology and physical ... [6] Positive psycho therapy in abi rehab (PoPsTAR): A pilot ... [7] Positive psychotherapy: A strength-based ... [8] Positive psychotherapy; Encyclopedia of quality ... [9] Positive psychology: An approach to supporting recovery ... [10] Positive group psychotherapy modified for adults with intellectual ... [11] Positive psychotherapy for smoking cessation: Treatment ... [12] Psychotherapy 2.0: Where psychotherapy ... [13] The perception of digital objects and their ... [14] Usefulness of telepsychiatry: A critical ... [15] A comprehensive review and a meta-analysis ... [16] A comparative cost analysis of an integrated ... [17] An exploratory study of the cyberbullying and ... [18] New mourners, old mourners: Online memorial ... [19] The effect of social network and short messages through e-content on ... [20] Impact of a mobile phone and web program on symptom and ... [21] Reliability and Validity of the Beck Depression ... [22] Psychometric properties of a Persian-language ... [23] Psychometric properties and measurement equivalence ... [24] Validation of Iranian version of pregnancy ... [25] Positive psychology: The science of happiness and human ... [26] Advantages and limitations of Internet-based ... [27] Internet and computer-based cognitive behavioral ... [28] Economic evaluations of Internet interventions for mental ... [29] Effects of an Internet intervention (Deprexis) on severe ... [30] Positive imagery-based cognitive bias modification as a web-based treatment tool for depressed adults ... [31] Randomized controlled trial of SuperBetter, a smartphone-based/Internet-based self-help tool ... [32] Guided Internet-delivered cognitive behavioural treatment for insomnia ... [33] Internetbased interventions for posttraumatic stress: A meta-analysis ... 
با محدوديتهاى جسمى ارايه مىكنند، مىتوانند موجب بهزيستى

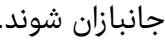

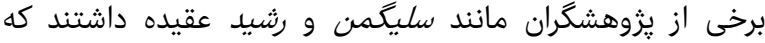

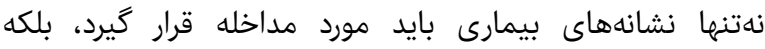

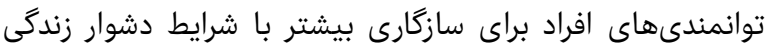

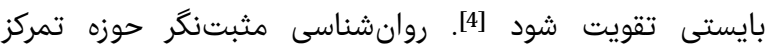

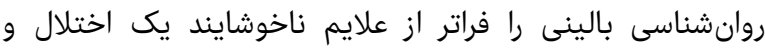

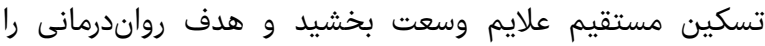

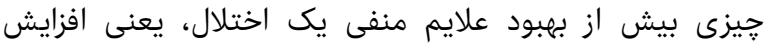

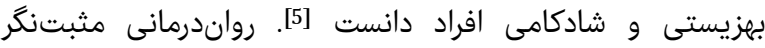

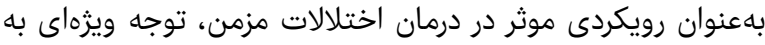

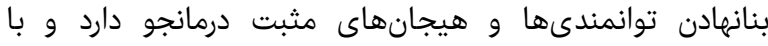

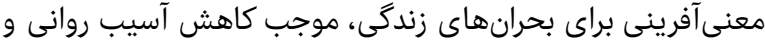

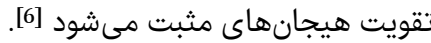

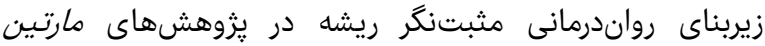

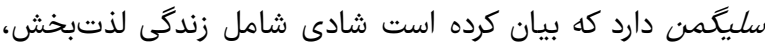

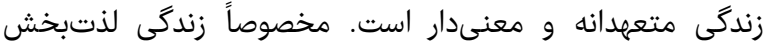

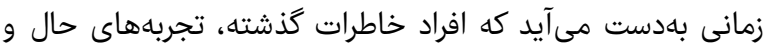

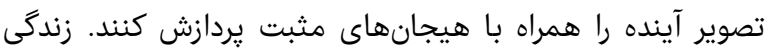

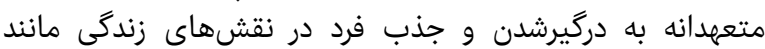

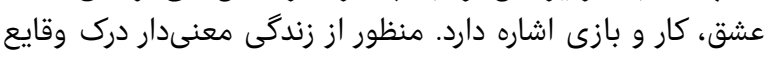

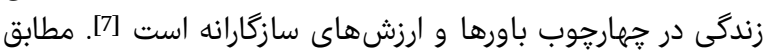

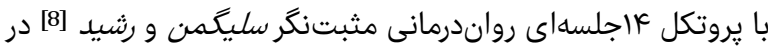

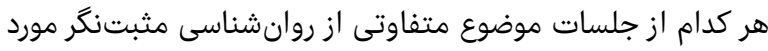

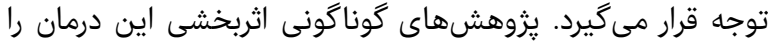

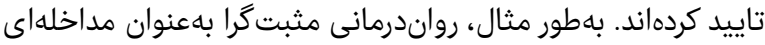

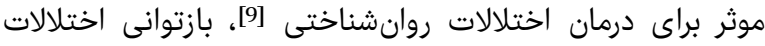

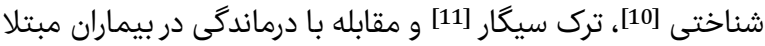

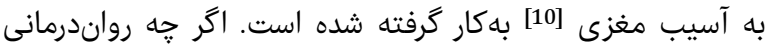

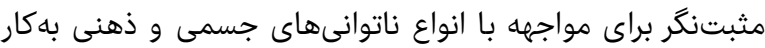

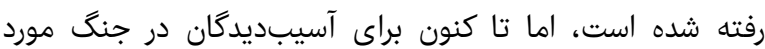

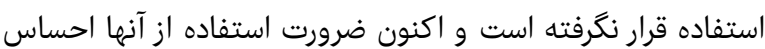

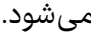

انقلاب اينترنت، زندكى بشر را كاملاً متحول ساخته است. اين

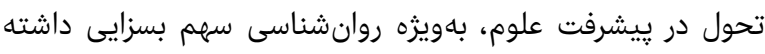

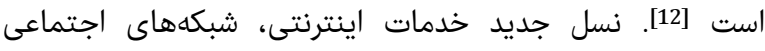

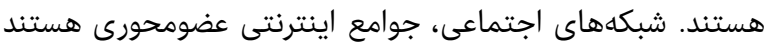

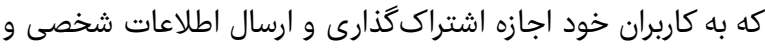

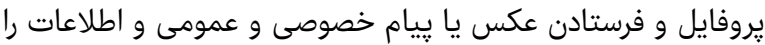

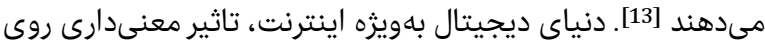

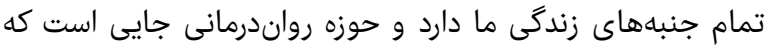

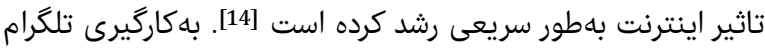

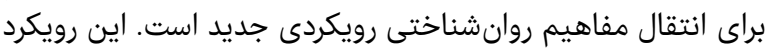

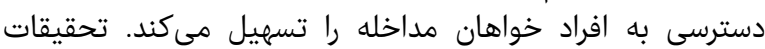

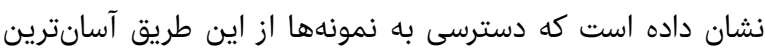

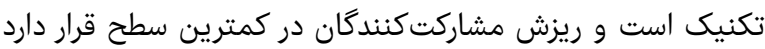

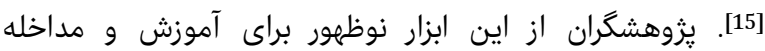

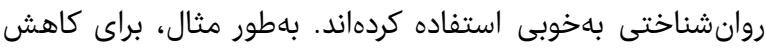

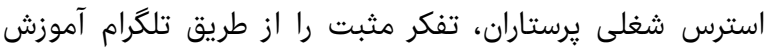

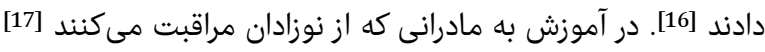

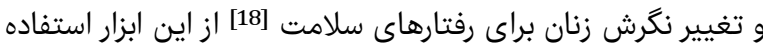

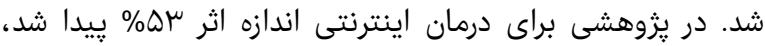

دوره •l، شماره ا، زمستان عوسا
تاثير رواندرمانى مثبت كراى مبتنى بر شبكههاى

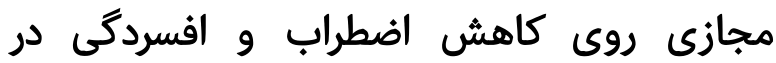

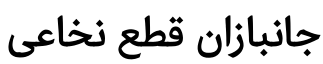

PhD " منيزه فيروزى كروه روان شناسى بالينى، يرديس فاروابى، دانشكاه تهران، تهران، ايران

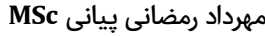
كروه روان شناسى بِالينى، يرديس فارابى، دانشكاه تهران، تهران، ايران

جكيده

اهداف: مشكلات جانبازان قطع نخاع، بهطور قابل ملاحظهاى سلامت روان إن-

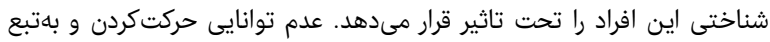

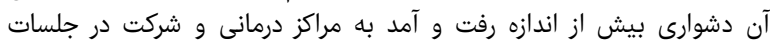

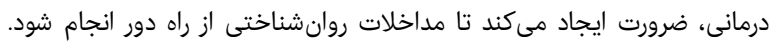

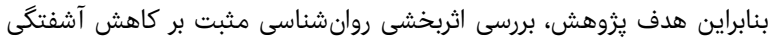

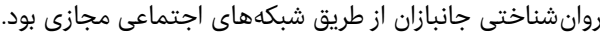

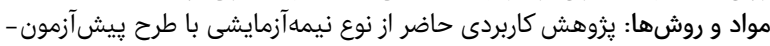

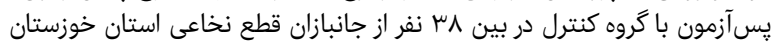

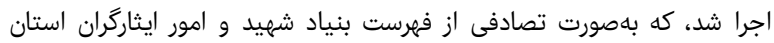

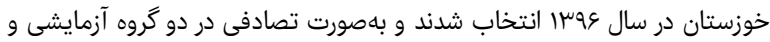

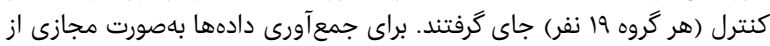

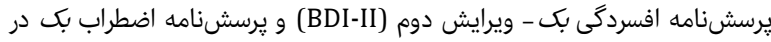

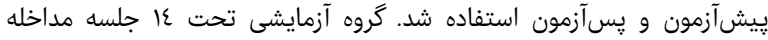

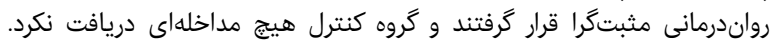

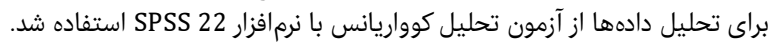

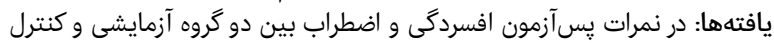

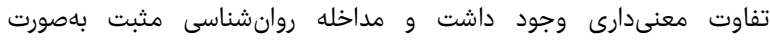

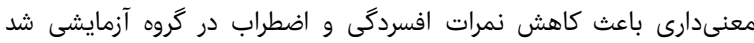
$(\mathrm{p}=\cdot / \cdot .1)$

نتيجهَيرى: مداخلات روانشناسى مثبتگراى مبتنى بر شبكه مجازى (تلكَرام)

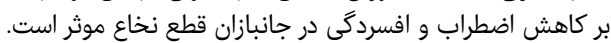

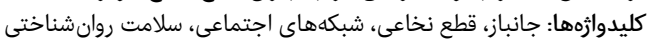

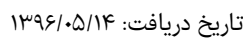

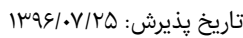

mfiroozy@ut.ac.ir : نويسنده مسئون:

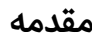

جنگ عوارض جبران نايديرى در ابعاد مختلف به بار مىآورد. يكى از

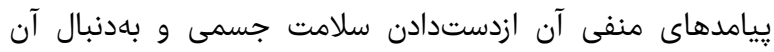

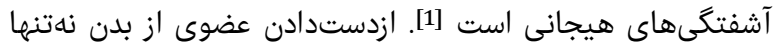

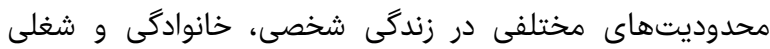

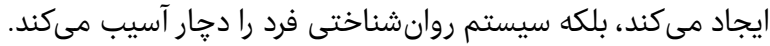

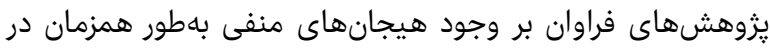

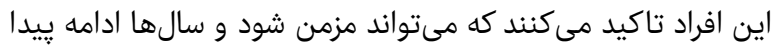

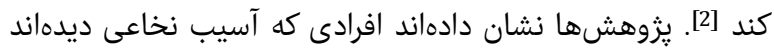

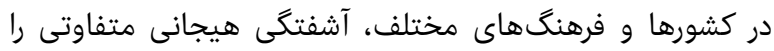

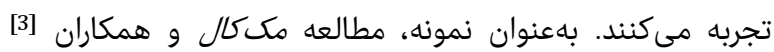

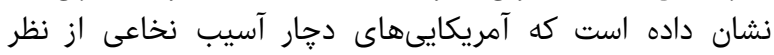

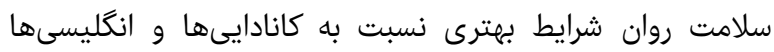

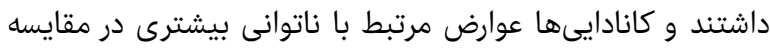

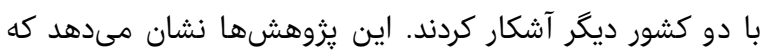

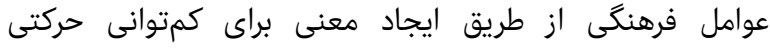

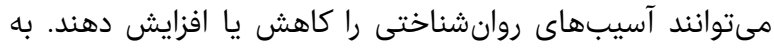

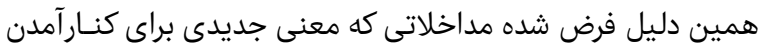

فصل نامه علمى - يُزوهشى طب جانباز 


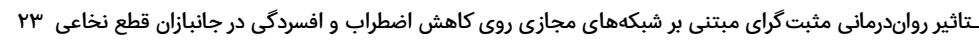

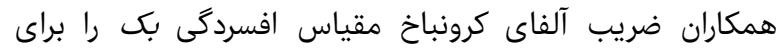

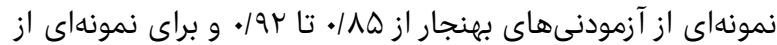

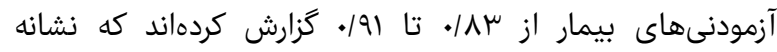

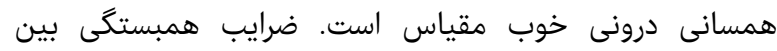

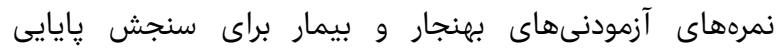

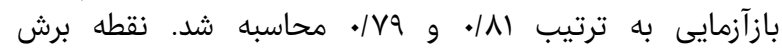

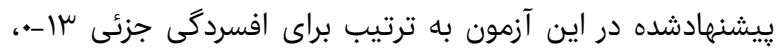

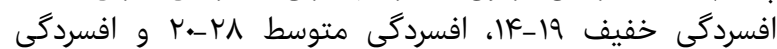

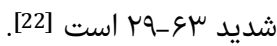

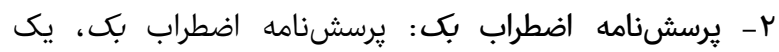

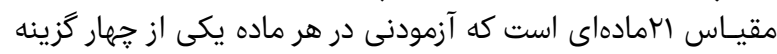

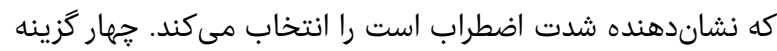

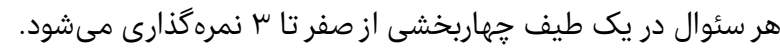

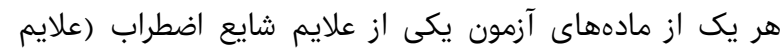

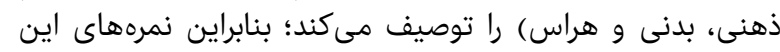

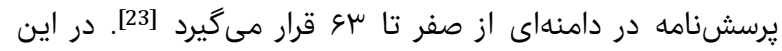

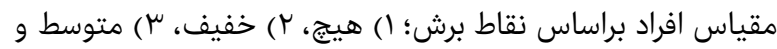

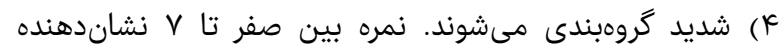

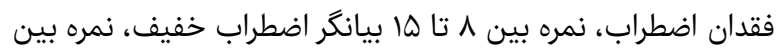

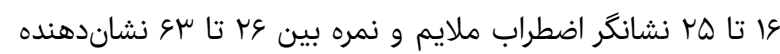

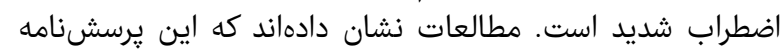

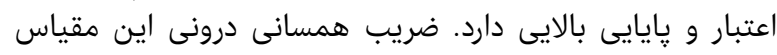

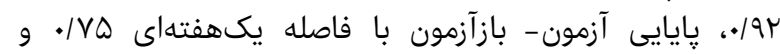

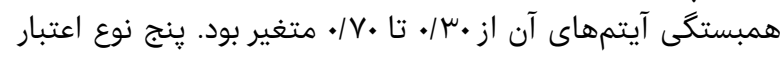

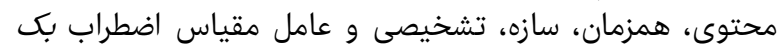

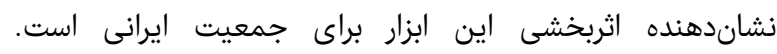

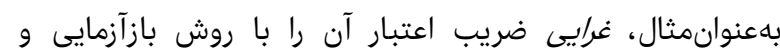

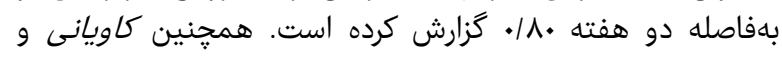

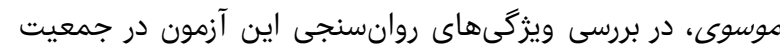

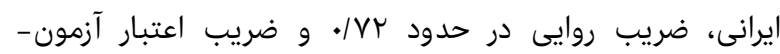

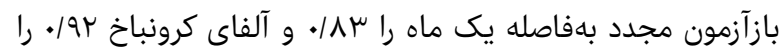

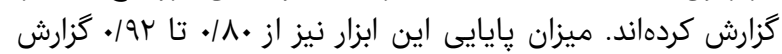

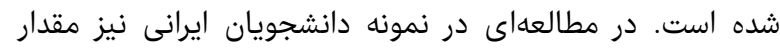

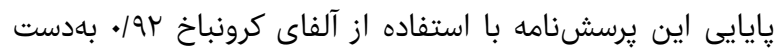

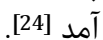

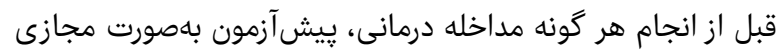

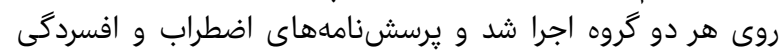

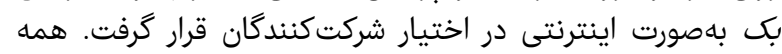

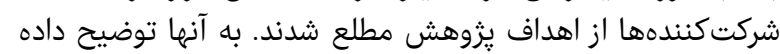

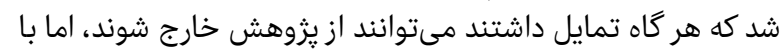

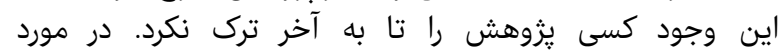

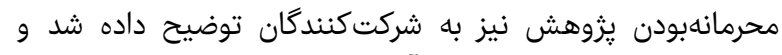

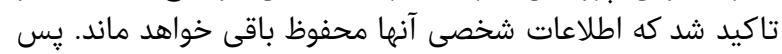

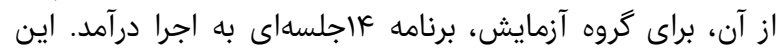

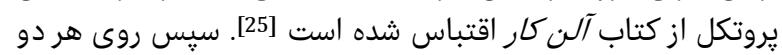

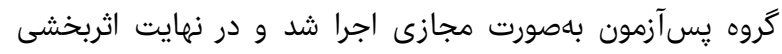

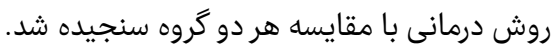

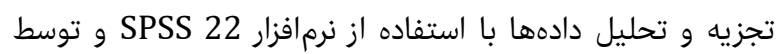

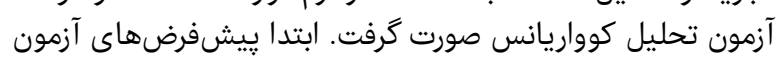

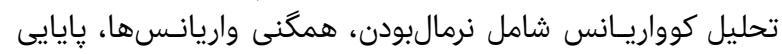

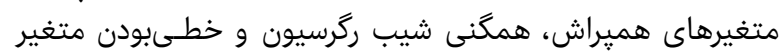

يعنى اثر رواندرمانى اينترنتى متوسط كزارش شد كه كاملاً شبيه

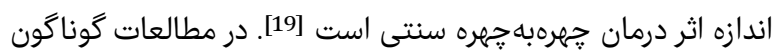

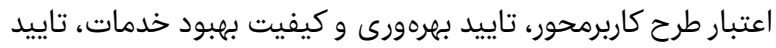

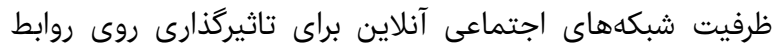

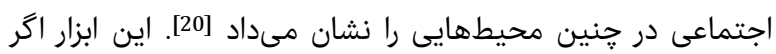

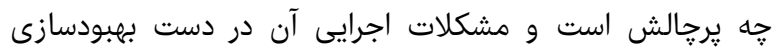
است، اما براى كسانى كه امكان دسترسى به به امكانات دروات درمانى ندارند،

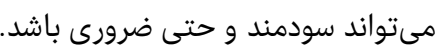

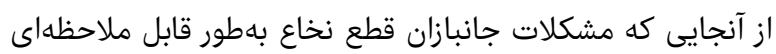

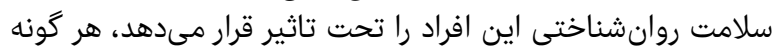

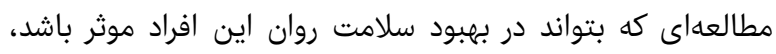

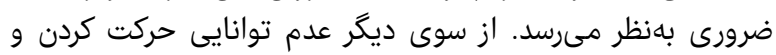

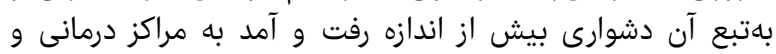

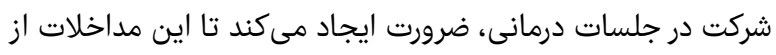

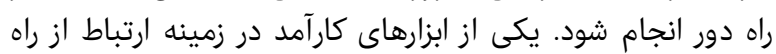

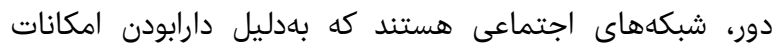

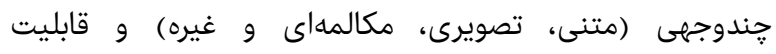
دردسترسبودن، مناسبتر از ساير وسايل ارتباط از از راه دور بهانظئ

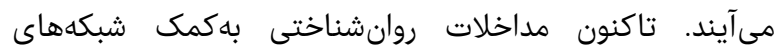

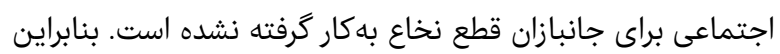

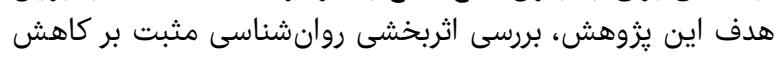

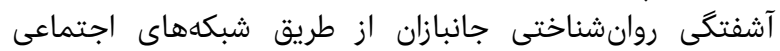

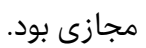

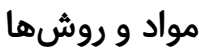

يزوهش كاربردى حاضر با روش نيمها آزمايشى از نوع ييشآ آزمون -

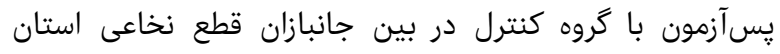

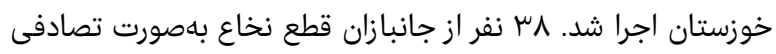

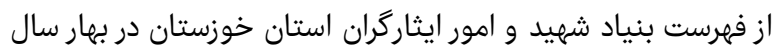

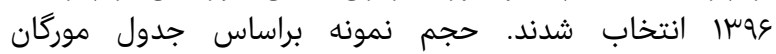

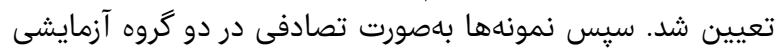

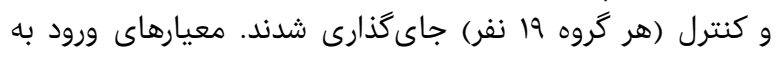

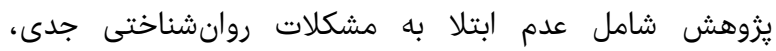

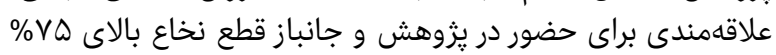

براى سنجش آشفتگى هيجانى از يرسشنامهنهاى زير استفاده شد:

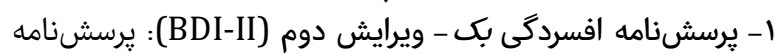

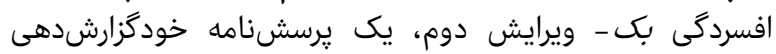

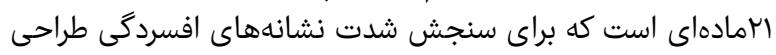

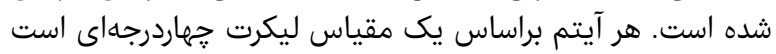

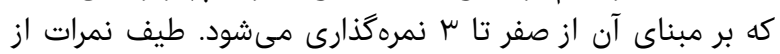

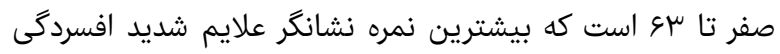

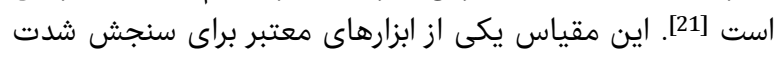

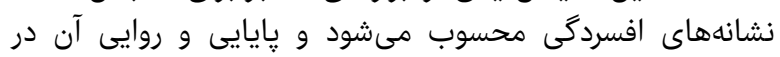

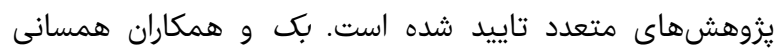

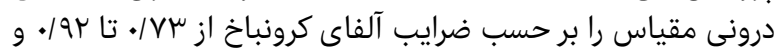

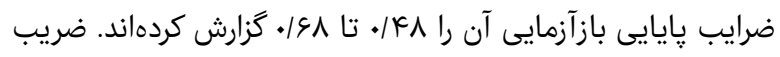

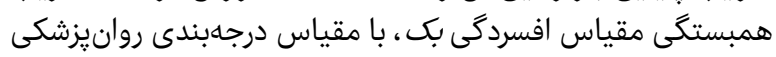

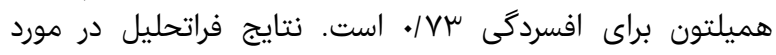

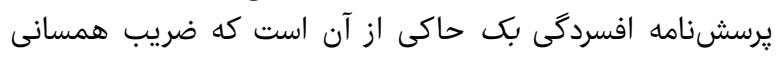

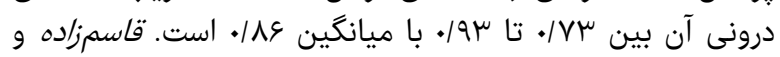


برنامه مداخلهاى بود نيز مىتوانست در كاهش اضطراب كارآمد

باشد.

جانبازان نسبت به عموم افراد با مشكلات روانى بيشترى دركيرند و آنان

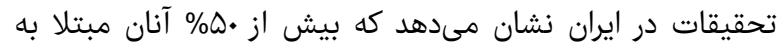

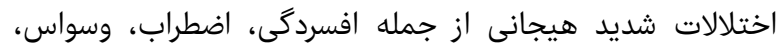

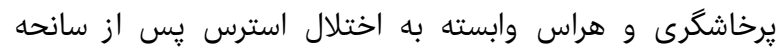

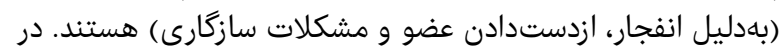

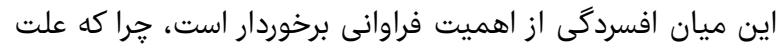

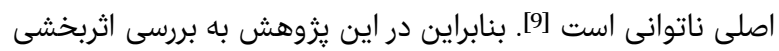

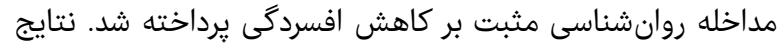

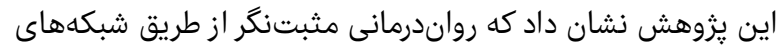

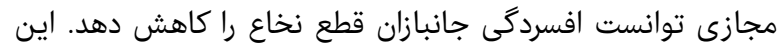

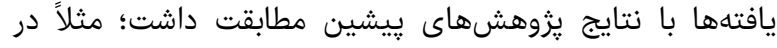

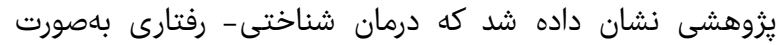

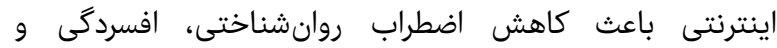

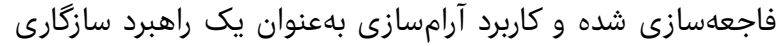

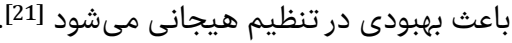

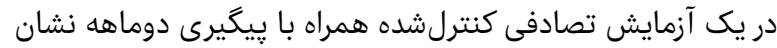

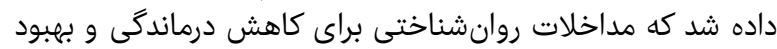

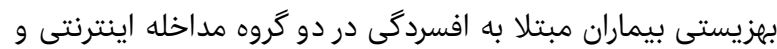

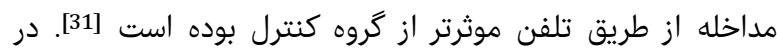

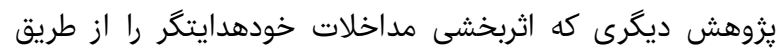

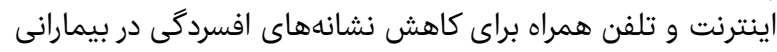

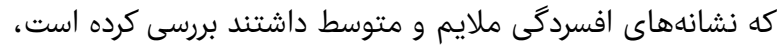

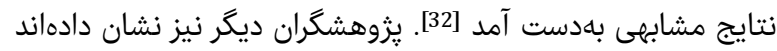

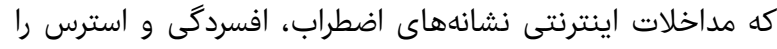

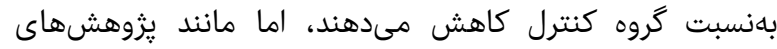

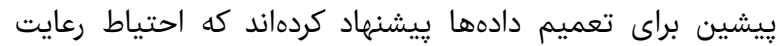

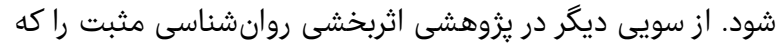

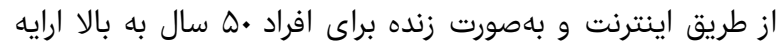

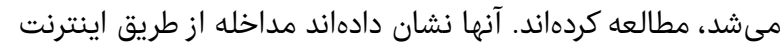

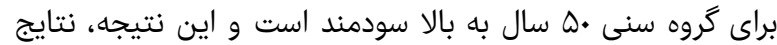

بهدستآمده در اين يزوهش •ـ سئ رال تاييد كرد [33].

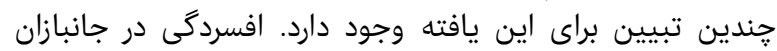

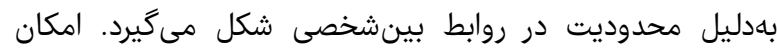

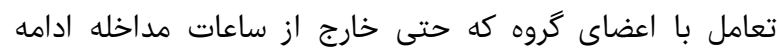

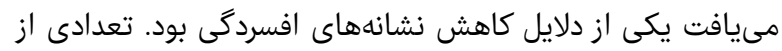

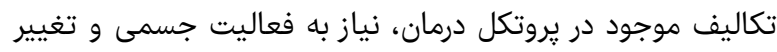

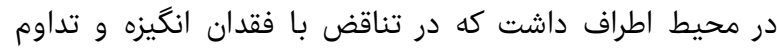

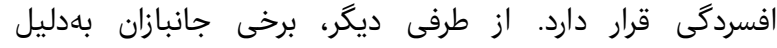

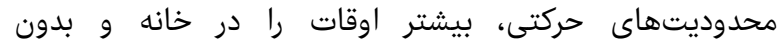

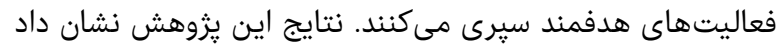

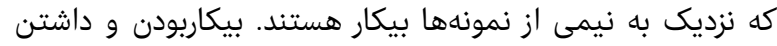

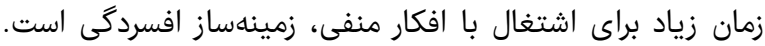

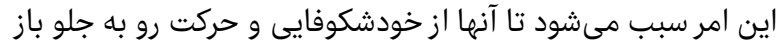

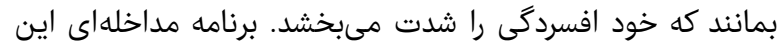

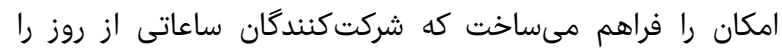

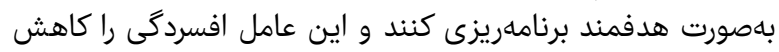

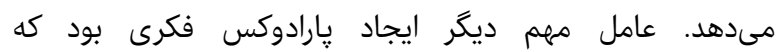

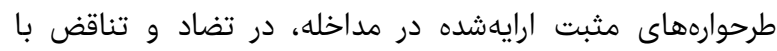

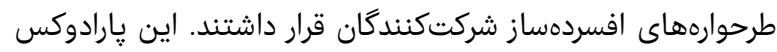

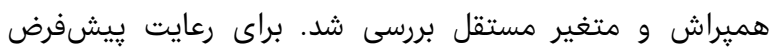

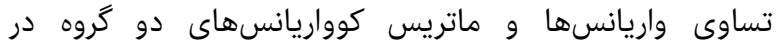

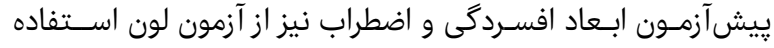

\section{يافتهها}

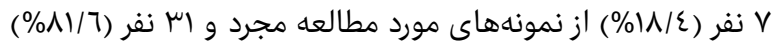

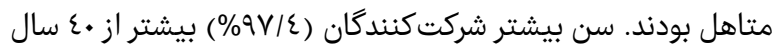

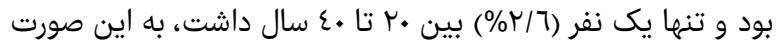

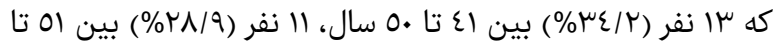

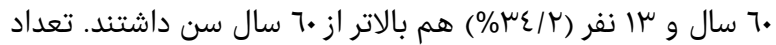

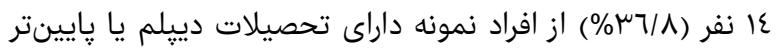

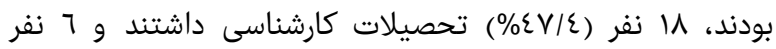

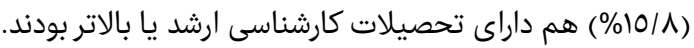

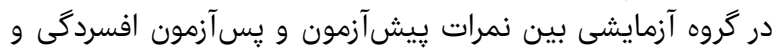

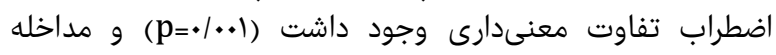

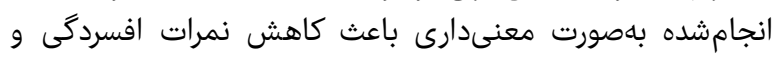

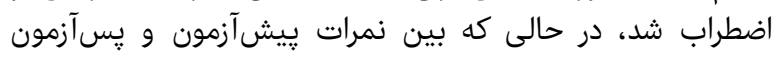

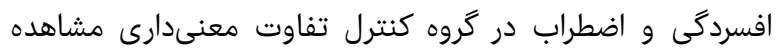

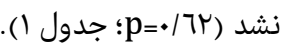

جدول () مقايسه ميانكين آمارى نمرات افسردگى و اضطراب دو گروه آزمايش و

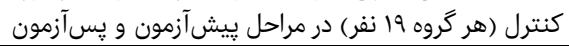

\begin{tabular}{|c|c|c|}
\hline كروه كنترل & كروه آزمايشى & متغيرها \\
\hline$r \wedge / Q \cdot \pm \mu / V \mu$ & $r V / . . \pm \mu / T V$ & 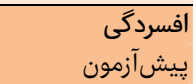 \\
\hline$r N / V \cdot \pm 0 / \cdot 1$ & $|V / O \cdot \pm \varepsilon / N|$ & يس آزمون \\
\hline$r V / 7 \cdot \pm \varepsilon / V \wedge$ & $r V / 0 \varepsilon \pm \varepsilon / \varepsilon \mid$ & اضيشرآ آزمون \\
\hline$r \Lambda / 1 \cdot \pm \varepsilon / l r$ & $r \mu / \cdot \mu_{ \pm} \mu / r \varepsilon$ & يسآزمون \\
\hline
\end{tabular}

\section{بحث}

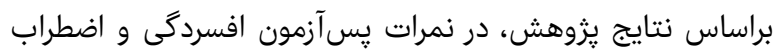

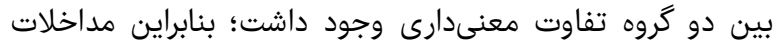

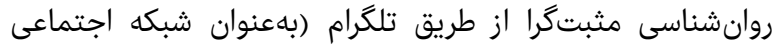
مدرن) بر كاهش اضطراب و افسردگى جانبازان قطع نخاع تاثير (بران

داشت.

طبق يافتهها، بين دو گروه آزمايشى و كنترل پِ إن از انجام مداخله

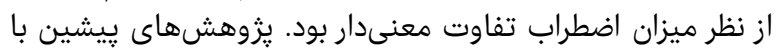

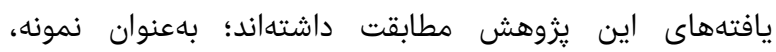

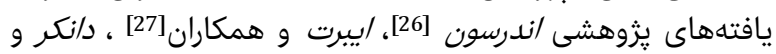

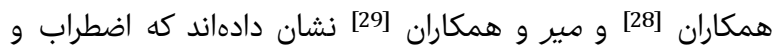

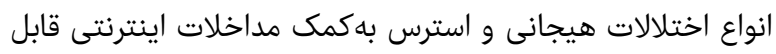

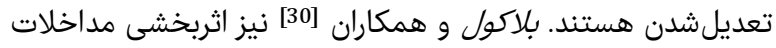

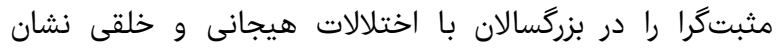

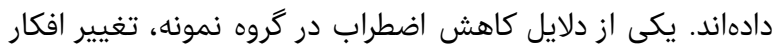

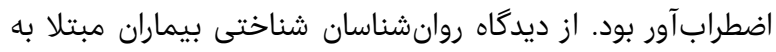

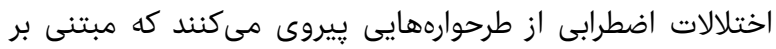

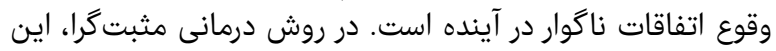

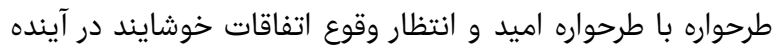

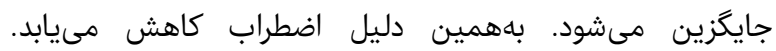

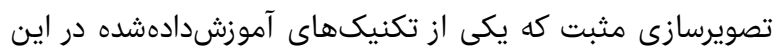


منابع

1- Khajavi D, MirAli H. Predicting trait competitive anxiety, competitive aggression, and competitive anger based on spiritual intelligence in veterans and disabled athletes. Iran J War Public Health. 2017;9(3):163-8.

2- Mikaeili N, Molavi P, Einy S, Tagavy M. Effectiveness of emotion focused therapy on emotional dysregulation, hopelessness and suicidal ideation in post-traumatic stress disorder veterans. Iran J War Public Health. 2017;9(3):112-7.

3- McColl MA, Charlifue S, Glass C, Savic G, Meehan M. International differences in ageing and spinal cord injury. Spinal cord. 2002;40(3):128-36.

4- Seligman MEP, Rashid T, Parks AC. Positive psychotherapy. Am Psychol. 2006;61(8):774-88.

5- Park N, Peterson Ch, Szvarca D. Positive psychology and physical health. Res Appl. 2014;10(3):200-6.

6- Cullen B, Pownall J, Cummings J, Baylan S, Broomfield $\mathrm{N}$, Haig $\mathrm{C}$, et al. Positive psycho therapy in abi rehab (PoPsTAR): A pilot randomised controlled trial. Neuropsychol Rehabil. 2016;4:1-17.

7- Rashid T. Positive psychotherapy: A strength-based approach. J Posit Psychol. 2015;10(1):25-40.

8- Rashid T. Positive psychotherapy; Encyclopedia of quality of life and well-being research. Netherlands: Springer; 2014. pp. 4933-8.

9- Schrank B, Brownell T, Tylee A, Slade M. Positive psychology: An approach to supporting recovery in mental illness. East Asian Arch Psychiatry. 2014;24(3):95-103.

10- Tomasulo DJ. Positive group psychotherapy modified for adults with intellectual disabilities. J Intellect Disabil. 2014;18(4):337-50.

11- Kahler ChW, Spillane NS, Day A, Clerkin EM, Parks A, Leventhal AM, et al. Positive psychotherapy for smoking cessation: Treatment development, feasibility, and preliminary results. J Posit Psychol. 2014;9(1):19-29.

12- Weitz P. Psychotherapy 2.0: Where psychotherapy and technology meet. London: Karnac Books; 2014.

13- Graham R. The perception of digital objects and their impact on development. J Psychoanal Psychother. 2013;27(4):269-79.

14- Chakrabarti S. Usefulness of telepsychiatry: A critical evaluation of videoconferencing-based approaches. World J Psychiatry. 2015;5(3):286-304.

15- Barak A, Hen L, Boniel Nissim M, Shapira NA. A comprehensive review and a meta-analysis of the effectiveness of internet-based psychotherapeutic interventions. J Technol Hum Serv. 2008;26(2-4):109-60. 16- Grady BJ. A comparative cost analysis of an integrated military telemental health-care service. Telemed J E-Health. 2002;8(3):293-300.

17- Kraft E, Wang J. An exploratory study of the cyberbullying and cyberstalking experiences and factors related to victimization of students at a public liberal arts college. Int J Techno. 2010;1(4):18.

18- Walter T. New mourners, old mourners: Online memorial culture as a chapter in the history of mourning. New Rev Hypermed Multimed. 2015;21(1-2):10-24.

19- Razmgah P, Mojtahedzadeh R, Borjaliloo S, Mohammadi A. The effect of social network and short messages through e-content on reducing negative thoughts in women. Virtual Learn Med Sci. 2016;7(4):e11160.

20- Proudfoot J, Clarke J, Birch MR, Whitton AE, Parker G, Manicavasagar $\mathrm{V}$, et al. Impact of a mobile phone and web program on symptom and functional outcomes for
عاملى براى تغيير شد. لازم به ذكر است شركت دكنندگان كسانى

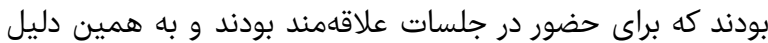
براى تغيير، انكيزه بالاترى داشتند.

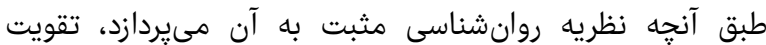

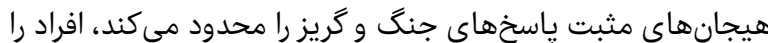

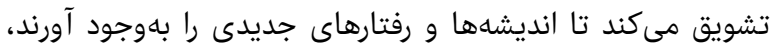

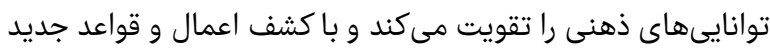

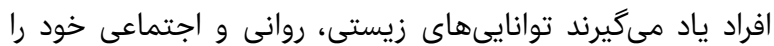

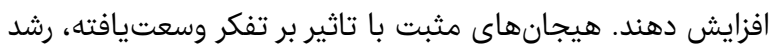

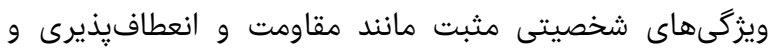

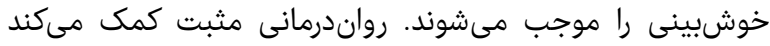

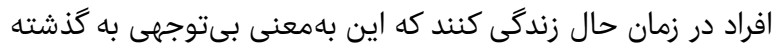

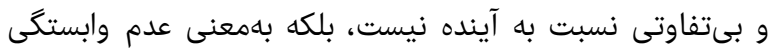

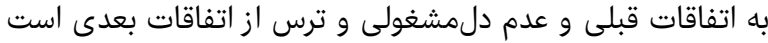

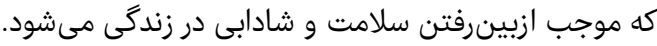

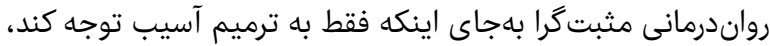

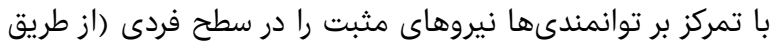

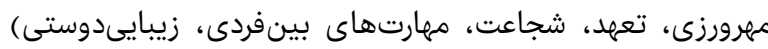

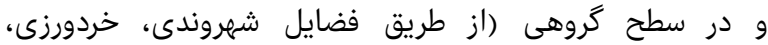

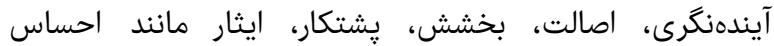

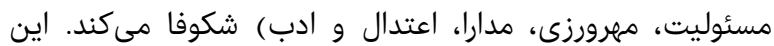

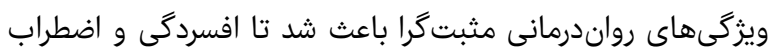

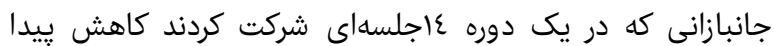

كند.

از محدوديتهاى اين مطالعه مىتوان به كنترلنكردن همه

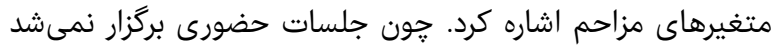

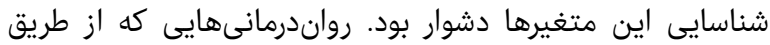

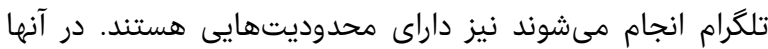

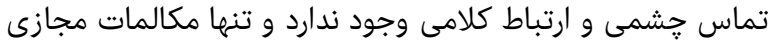

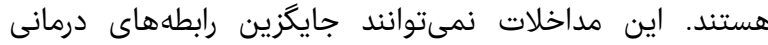

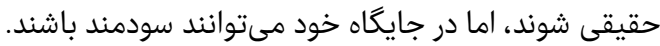

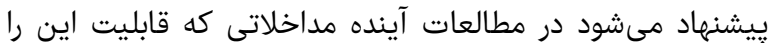

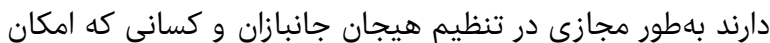

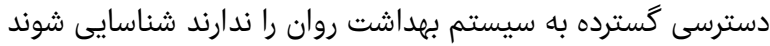
و اثربخشى آنها مورد مطالعه قرار گيرد.

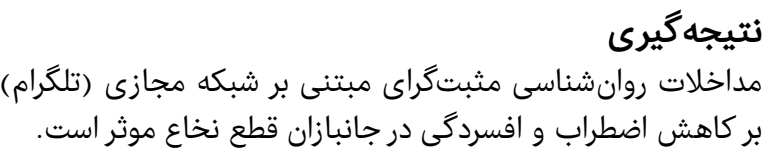

تشكر و قدردانى: از جانبازان استان خوزستان كه در اين يزوهش با

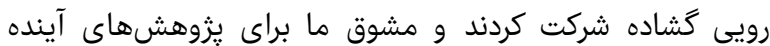
بودند، صميمانه تشكر مئكنيم.

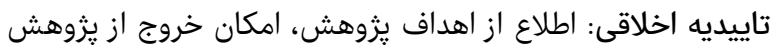

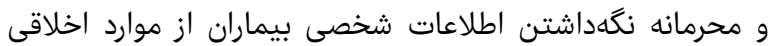

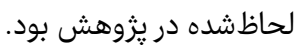

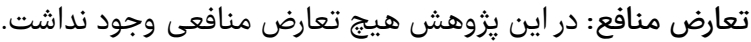

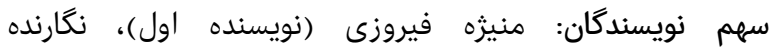

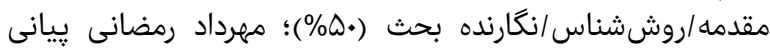

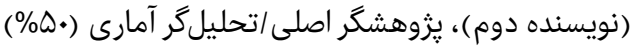

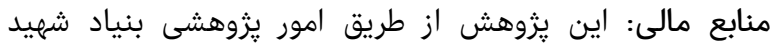

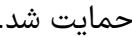


cognitive behavioral therapy for anxiety and depression in youth: A meta-analysis of randomized controlled outcome trials. PloS One. 2015;10(3): e0119895.

28- Donker T, Blankers M, Hedman E, Ljotsson B, Petrie $\mathrm{K}$, Christensen H. Economic evaluations of Internet interventions for mental health: A systematic review. Psychol Med. 2015;45(16):3357-76.

29- Meyer B, Bierbrodt J, Schröder J, Berger Th, Beevers CG, Weiss M, et al. Effects of an Internet intervention (Deprexis) on severe depression symptoms: Randomized controlled trial. Internet Int. 2015;2(1):48-59.

30- Blackwell SE, Browning M, Mathews A, Pictet A, Welch J, Davies J, et al. Positive imagery-based cognitive bias modification as a web-based treatment tool for depressed adults: A randomized controlled trial. Clin Psychol Sci. 2015;3(1):91-111.

31- Roepke AM, Jaffee SR, Riffle OM, McGonigal J, Broome R, Maxwell B. Randomized controlled trial of SuperBetter, a smartphone-based/Internet-based selfhelp tool to reduce depressive symptoms. Games Health J. 2015;4(3):235-46.

32- Van Straten A, Emmelkamp J, De Wit J, Lancee J, Andersson G, van Someren EJ, et al. Guided Internetdelivered cognitive behavioural treatment for insomnia: A randomized trial. Psychol. Med. 2014;44(7):1521-32. 33- Kuester A, Niemeyer H, Knaevelsrud C. Internetbased interventions for posttraumatic stress: A metaanalysis of randomized controlled trials. Clin Psychol Rev. 2016;43:1-16. stress: A randomised controlled trial. BMC Psychiatry. 2013;13:312.

21- Lee EH, Lee SJ, Hwang ST, Hong SH, Kim JH. Reliability and Validity of the Beck Depression Inventory-II among Korean Adolescents. Psychiatry Investig. 2017;14(1):30-6.

22- Ghassemzadeh H, Mojtabai R, Karamghadiri N, Ebr-

ahimkhani N. Psychometric properties of a Persian-language version of the Beck Depression Inventory-Second edition: BDI-II-Persian. Depress Anxiety. 2005;21(4):185-92.

23- Ke Y, Ng T, Yeo HL, Shwe M, Gan YX, Chan A. Psychometric properties and measurement equivalence of the English and Chinese versions of the Beck Anxiety Inventory in patients with breast cancer. Support Care Cancer. 2017;25(2):633-43.

24-Askarizadeh G, Karamoozian M, Darekordi A. Validation of Iranian version of pregnancy related anxiety questionnaire. Int J Prev Med. 2017;8:17.

25- Carr A. Positive psychology: The science of happiness and human strengths. Abingdon-on-Thames: Routledge; 2011.

26- Andersson G, Titov N. Advantages and limitations of Internet-based interventions for common mental disorders. World Psychiatry. 2014;13(1):4-11

27- Ebert DD, Zarski AC, Christensen H, Stikkelbroek Y, Cuijpers $\mathrm{P}$, Berking $\mathrm{M}$, et al. Internet and computer-based 